PROFESIONALES Y HERRAMIENTAS PARA EL DESARROLLO LOCAL Y SUS SINERGIAS TERRITORIALES. EVALUACIÓN Y PROPUESTAS DE FUTURO IX Coloquio Nacional de Desarrollo Local del GTDL-AGE 

ANTONIO MARTÍNEZ PUCHE, XAVIER AMAT MONTESINOS, ISABEL SANCHO CARBONELL y DANIEL SANCHIZ CASTAÑO (EDS.)

\section{PROFESIONALES Y HERRAMIENTAS PARA EL DESARROLLO LOCAL Y SUS SINERGIAS TERRITORIALES. EVALUACIÓN Y PROPUESTAS DE FUTURO}

IX Coloquio Nacional de Desarrollo Local del GTDL-AGE

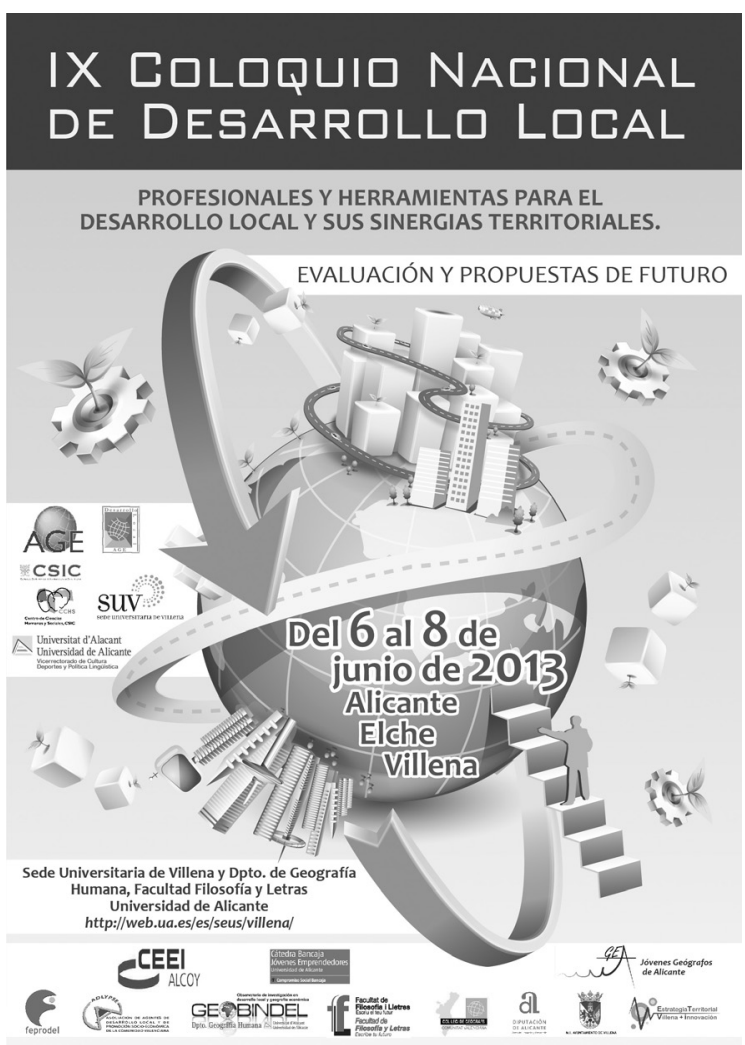


Este libro ha sido examinado y valorado por evaluadores ajenos a la Universidad de Alicante, con el fin de garantizar la calidad científica del mismo.

Publicacions de la Universitat d'Alacant

03690 Sant Vicent del Raspeig

Publicaciones@ua.es

http://publicaciones.ua.es

Telèfon: 965903480

(C) Antonio Martínez Puche, Xavier Amat Montesinos,

Isabel Sancho Carbonell y Daniel Sanchiz Castaño (eds.), 2016

(C) d'aquesta edició: Universitat d'Alacant

ISBN: 978-84-16724-00-0

Dipòsit legal: A 92-2016

Disseny de coberta: candela ink

Composició: Página Maestra (Miguel Ángel Sánchez Hernández)

Impressió i enquadernació: Guada Impresores

\section{unte \\ Unión de Editoriales
Universitarias Españolas \\ WWW.une.es
WWA}

Esta editorial es miembro de la UNE, cosa que garantiza la difusión y comercialización nacional y internacional de sus publicaciones.

Reservados todos los derechos. Cualquier forma de reproducción, distribución, comunicación pública o transformación de esta obra sólo puede ser realizada con la autorización de sus titulares, salvo excepción prevista por la ley. Diríjase a CEDRO (Centro Español de Derechos Repográficos, www.cedro.org) si necesita fotocopias o escanear algún fragmento de esta obra. 


\title{
PROPUESTA PARA LA DIVERSIFICACIÓN PARA LA DIVERSIFICACIÓN SOCIOECONÓMICA DE SANT JOAN D'ALACANT: APROVECHAMIENTO DE LOS RECURSOS TERRITORIALES DE OCIO
}

\author{
Vanessa Romá Beneyto \\ Alumna de Doctorado de Desarrollo Local e Innovación Territorial. Universidad de Alicante \\ Vanessarb77@hotmail.com
}

RESUMEN

Sant Joan es un municipio de interior con una fuerte tradición agrícola, ubicado en pleno corazón de la desaparecida Huerta de Alicante. Posee un importante legado patrimonial enmarcado en diferentes épocas, que necesita un plan de gestión que establezca estrategias centradas en la cultura, la innovación y el desarrollo socioeconómico. Permitiría potenciar la oferta turística basada en el ocio de proximidad y la sostenibilidad, y a su vez recuperar y proteger los monumentos declarados Bien de Interés Cultural que han sido expoliados, abandonados y olvidados, y que actualmente se encuentran en un estado ruinoso.

Palabras clave: Desarrollo socioeconómico local, Crecimiento Integral, Nuevas Tecnologías, Centro de Interpretación, Patrimonio Cultural.

\section{PROPOSAL FOR A SOCIO-ECONOMICO DIFERSIFICATION OF SAN JUAN DE ALICANTE: USE OF TERRITORIAL LEISURE RESOURCES}

AbSTRACT

Situated at the heart of an area formerly known as La Huerta de Alicante, San Juan is an inland city with a strong agricultural tradition. Its very wealthy heritage, coming from different epochs, should be administered by a strategical plan based on culture, innovation and socio-economic development. It would promote a type of tourism based on leisure of proximity and sustainability as well as restore and protect historically classified monuments which have been ransacked, neglected and are, at present, in a bad condition. 
Key Words: Local socio-economic development, Endogenous development, New Tecnologies, Cultural Heritage Centre, Cultural Heritage.

\section{JUSTIFICACIÓN}

Sant Joan es un pequeño municipio que cuenta con un extenso catálogo patrimonial e histórico enmarcado en diferentes épocas. Sus actividades principales son los servicios y el turismo residencial. Se encuentra situado a tan sólo un kilómetro de la playa, pero es considerado municipio de interior.

Para aprovechar las riquezas del municipio, necesitamos diseñar estrategias centradas en la cultura, la innovación y el desarrollo socioeconómico, a través de una oferta turística basada en el ocio de proximidad y la sostenibilidad.

El proyecto planteado pretende crear un Centro de Interpretación que servirá de herramienta para recuperar y proteger la mayor parte de los monumentos declarados Bien de Interés Cultural que han sido expoliados, abandonados y olvidados, y que se encuentran actualmente en ruinas. Será también una forma de trasmitir este Patrimonio Cultural Inmaterial a la población y potenciar la oferta cultural para el turismo.

Sant Joan es un claro ejemplo de municipio pequeño con un importante legado patrimonial que necesita un plan de gestión y del cual el municipio podrá extraer una fuente de ingresos y de revaloración continua.

\section{OBJETIVOS}

Se pretende alcanzar un crecimiento integral, endógeno y controlado del territorio, con análisis de recursos particulares y específicos del municipio, basándonos en cuatro funciones básicas: investigación, conservación, divulgación y puesta en valor del patrimonio. Para alcanzar este propósito, se propone el siguiente objetivo principal:

La apertura de un Centro de Interpretación reforzará el interés cultural y situará el municipio como destino turístico emergente, gracias a elementos y acciones que pongan en valor nuestro patrimonio y contribuirá a su vez al desarrollo sostenible del municipio. Para ello, se van a implementar los siguientes objetivos específicos:

- Identificar los elementos del patrimonio con un interés cultural y así definir cuál es su verdadera seña de identidad y la estructura de su patrimonio histórico.

- Establecer diferentes ejes para el desarrollo sostenible del municipio.

- Involucrar a la población local en el proyecto mediante foros participativos y generar nuevas iniciativas.

- Estudiar el impacto de la creación del Centro de Interpretación en la población local. 
- Fomentar la conciencia cultural a través de proyectos y talleres didácticos con el objetivo de dar a conocer la importancia de la Huerta de Alicante durante muchos siglos.

Todos estos objetivos justifican su razón de ser pero hay un aspecto que queremos resaltar: el deseo de no perder el contacto con las generaciones que nos precedieron. Pensamos con toda firmeza que la herencia, tradiciones y costumbres de nuestros antepasados forman los pilares de nuestra sociedad y son esenciales para consolidar nuestra identidad y la de las futuras generaciones.

El Centro de Interpretación pretende ser un espacio donde se pueda apreciar el patrimonio tangible e intangible. A través de documentos gráficos, vídeos y la incorporación de las nuevas tecnologías, el visitante podrá descubrir la cultura, las fiestas, la gastronomía, los monumentos y la tradición a lo largo de la historia, vivir experiencias irrepetibles y descubrir el legado histórico-cultural que posee el municipio de Sant Joan. Si conocemos y respetamos el patrimonio cultural del municipio ayudaremos a las generaciones futuras a conservarlo y cuidarlo.

En resumen, este proyecto surge por el afán despertado al descubrir la necesidad de valorar, recuperar y conservar esa parte de la historia, la importancia del territorio, que constituye señas de identidad olvidadas por unos pocos y desconocida por muchos. Por ello, se realiza un análisis descriptivo de la situación actual del municipio, de la evolución y tendencias para conocer las expectativas y necesidades demandadas por la población local, que se presenta de una forma resumida en este documento.

\section{MARCO TEÓRICO}

Sant Joan cuenta con una serie de patrimonio histórico cultural que forma parte de la desaparecida Huerta de Alicante. El abandono, la crisis agrícola y los nuevos usos del suelo han dado lugar a este proceso. En este contexto, surge la necesidad de recuperación y conservación y se plantea la posibilidad de crear un Centro que ponga en valor el patrimonio, y se puedan llevar a cabo los objetivos anteriormente mencionados.

La Unesco define Patrimonio Cultural como «el conjunto de bienes muebles e inmuebles, materiales e inmateriales, de propiedad de particulares o de instituciones u organismos públicos o semipúblicos que tengan valor excepcional desde el punto de vista de la historia, del arte, de la ciencia y de la cultura y por lo tanto sean dignos de ser considerados y conservados para la nación» (Unesco, 1977).

«La interpretación, lejos de ser un fenómeno nuevo, es una de las practicas más antiguas de transferencia cultural (Uzzell, 1989), no así el termino interpretación, incorporado solo a partir de finales del siglo XIX» (Peart, 1980).

Teóricamente, el turismo se alimenta con la explotación de recursos renovables, para que el proceso de producción termine con la prestación y venta del producto final, lo que pone en peligro los recursos naturales (Molina, 1982). 
Moscardo y Pearce (1988) indican tres elementos fundamentales a tener en cuenta para un modelo de desarrollo óptimo:

1. Los recursos que un determinado lugar tiene para ofrecer.

2. Las necesidades de los habitantes locales

3. Las necesidades y expectativas de los visitantes.

Un requisito indispensable para cualquier actividad turística que pretenda tener éxito es la información. Los clientes potenciales deben saber qué oferta hay disponible y poseer la suficiente información para tener una base sobre la cual elegir los servicios turísticos (Moscardo y Pearce, 1988).

Es por ello que surge la necesidad de crear nuevas infraestructuras y modelos que se adapten a estos cambios y poder crear un destino cultural, eso es, no sólo tener patrimonio sino saber gestionarlo para conseguir buenos resultados.

En la actualidad, existen muchas exposiciones que por la mala gestión, la falta de contenido interesante o simplemente por la crisis que nos aborda, se ven condicionadas a un cierre inminente o un abandono casi absoluto. Por ello, es muy importante tener en cuenta aspectos tan importantes como el interés real del turista y cómo se le puede ofrecer un producto innovador, llamativo y de fácil acceso.

\subsection{Análisis DAFO}

Sant Joan cuenta con una buena cantidad de oportunidades para su desarrollo pero también con un gran número de amenazas. Para sintetizar todo ello, hemos realizado el siguiente análisis DAFO con el fin de presentar de una forma resumida cuáles son y cómo nos afectan directa e indirectamente para el desarrollo de nuestro proyecto:

\section{Cuadro 1. Análisis DAFO}

\begin{tabular}{|c|c|}
\hline $\begin{array}{l}\text { Fortalezas: } \\
\text { - Antigüedad de los monumentos. } \\
\text { - Monumentos catalogándolos como BIC y BRL. } \\
\text { - Monumentos típicos de la Huerta de Alicante. } \\
\text { - Protegidos en el PGOU y en el Catálogo de } \\
\text { Edificios Protegidos de Sant Joan d'Alacant. } \\
\text { - Restauración y puesta en valor por asociaciones } \\
\text { y entes politicos. }\end{array}$ & $\begin{array}{l}\text { Oportunidades: } \\
\text { - Interés creciente de la sociedad por el } \\
\text { patrimonio cultural. } \\
\text { - Interés por parte de las Universidades en temas } \\
\text { relacionados con el patrimonio histórico así } \\
\text { comotambién en la restauración. } \\
\text { - Aumento del interés por el turismo cultural con } \\
\text { gran demanda de productosy servicios. } \\
\text { - Próxima instalación de la UCAM. }\end{array}$ \\
\hline $\begin{array}{l}\text { Debilidades: } \\
\text { - Escasa potencialidad en el entomo, no existe } \\
\text { una oferta potencial que atraiga visitantes. } \\
\text { - Los bienes patrimoniales están alejados del } \\
\text { núcleo urbanoy sin senalización. } \\
\text { - Falta de promoción e información turística. } \\
\text {-Poca implicación de los habitantes del } \\
\text { municipio. } \\
\text { - Escaso uso de los bienes públicos. }\end{array}$ & $\begin{array}{l}\text { Amenazas: } \\
\text { - Crecimiento inmobiliario desordenado de escaso } \\
\text { respeto paisajistico } \\
\text { - Escasa valorización y concienciación social de } \\
\text { la importancia del patrimoniomunicipal. } \\
\text { - Falta de conservación y mantenimiento de los } \\
\text { bienes patrimoniales con la consiguiente pérdida } \\
\text { si no se lleva alguna acción pronto. } \\
\text { - La crisis actual. }\end{array}$ \\
\hline
\end{tabular}

Fuente: Elaboración propia 


\subsection{Técnica de investigación}

La finalidad de esta investigación es evaluar de forma individual la opinión de la población de Sant Joan y así conocer su visión e indagar en su percepción ante el nuevo proyecto de creación de un Centro de Interpretación del patrimonio del municipio. Para ello, se ha realizado una encuesta a individuos residentes en Sant Joan d'Alacant con el objetivo de profundizar en su percepción sobre el tema.

Para este estudio, se ha recogido información primaria a través de metodología cuantitativa, basada en la captación de información a través de una muestra representativa seleccionada entre la población de Sant Joan, por lo que los datos recogidos permiten su extrapolación a toda la población objeto de estudio. Se ha calculado el diseño muestral, adaptado a las características de la población objeto de estudio. La validez y representatividad de los resultados de esta encuesta, permite extrapolar los datos recogidos de los cuestionarios de 101 personas al conjunto de ciudadanos de Sant Joan d'Alacant.

Para determinar el tamaño de la población, la muestra, y llevar a cabo la distribución de la encuesta, se han utilizado los datos del Avance del «Padrón Municipal de Habitantes» del 1 de enero de $2011^{1}$ que presenta el Instituto Nacional de Estadística.

Una vez estratificada la población se ha realizado una afijación proporcional de la muestra: el número de cuestionarios cumplimentados en cada estrato depende proporcionalmente de su peso dentro de la distribución de la población por sexos, y del grupo de edad.

A continuación se presenta la Ficha Técnica de la encuesta realizada:

\section{Cuadro 2. Ficha técnica}

\begin{tabular}{|l|l|}
\hline \multicolumn{2}{|l|}{ FICHA TÉCNICA } \\
\hline UNIVERSO & Población de Sant Joan mayor de 18 años \\
\hline ÁMBITO GEOGRÁFICO & Municipio de Sant Joan d'Alacant \\
\hline TAMÃ̃O DE LA POBLACIÓN & 17.752 habitantes mayores de 18 años. \\
\hline NIVEL DE CONFIANZA & $95 \%$ \\
\hline ERROR MUESTRAL & $+/-9,25 \%$ \\
\hline TAMAÑ DE LA MUESTRA & 101 individuos \\
\hline TIPO DE MUESTREO & $\begin{array}{l}\text { Aleatorio Estratificado por sexo y edad -los casos } \\
\text { se han seleccionado de forma arbitraria- }\end{array}$ \\
\hline CRITERIOS DE AFIJACIÓN & Proporcional \\
\hline
\end{tabular}

1 Datos más actualizados a la hora de realizar el trabajo de campo. 


\begin{tabular}{|l|l|}
\hline $\begin{array}{l}\text { CRITERIOS DE ESTRATI- } \\
\text { FICACIÓN }\end{array}$ & $\begin{array}{l}\text { 1. Sexo } \\
\text { 2. Grandes grupos de edad (jóvenes, adultos, adul- } \\
\text { tos-mayores) }\end{array}$ \\
\hline $\begin{array}{l}\text { FECHA DE REALIZACIÓN } \\
\text { DEL TRABAJO DE CAMPO }\end{array}$ & 20 de marzo de 2012 - 30 de marzo 2012 \\
\hline
\end{tabular}

Fuente: Elaboración propia.

La muestra se ha llevado a cabo según las siguientes variables de estratificación: grandes grupos edad y sexo. A continuación se incluye la distribución muestral por grupos de edad y sexo.

\section{Cuadro 3. Diseño muestral}

\begin{tabular}{|c|c|c|c|}
\hline \multirow[b]{2}{*}{ GRUPOS DE EDAD } & \multicolumn{3}{|c|}{ SEXO } \\
\hline & $\dot{\mid i}$ & 穷 & TOTAL \\
\hline 18-34 A ̃̃OS & 15 & 14 & 29 \\
\hline 35-49 AÑOS & 16 & 16 & 32 \\
\hline 50-64 AÑOS & 9 & 12 & 21 \\
\hline 65 AÑOS Y MÁS & 9 & 10 & 19 \\
\hline TOTAL & 49 & 52 & 101 \\
\hline
\end{tabular}

Fuente: Elaboración propia.

\subsection{Análisis de las encuestas realizadas}

El turismo de interior debe apoyarse en experiencias para generar nuevas actividades. Si se quiere adecuar un espacio para albergar y dar a conocer la importancia patrimonial, hay que realizar un proceso de análisis y toma de decisiones para conseguir un buen resultado final, así como la «interpretación del patrimonio visitado» o lo que es lo mismo «que el público disfrute, aprecie y entienda fácilmente el significado de lo visitado» (Martínez Puche, et alii 2009).

Para alcanzar el sentimiento de pertenencia a un lugar es necesario aprender a valorar lo que rodea a cada uno, es decir, el contexto en el cual crecen las raíces que nos hacen de un lugar o de otro. En este aspecto, es imprescindible tomar conciencia de la importancia que tiene el legado cultural heredado de épocas pasadas, y tras esto, hacer cuanto esté en nuestra mano para salvaguardar y transmitir a las generaciones venideras tan rico tesoro.

\subsubsection{Grado de conocimiento del patrimonio}

Con el objetivo de indagar en el grado de conocimiento que tienen del patrimonio cultural del municipio, se cuestiona a los santjoaners si conocen los lugares 
de interés turístico y cultural que pueden visitar. Como vemos en el gráfico 1, siete de cada diez individuos encuestados responden afirmativamente.

En el gráfico 2, se cuestiona como se percibe la gestión de los recursos patrimoniales del municipio, obteniendo que el $74 \%$ aprecia que la gestión no es la correcta.

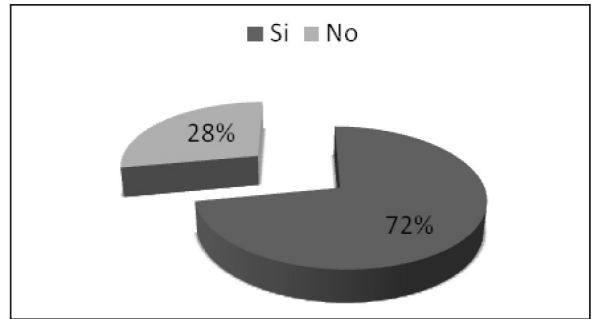

Gráfico 1. ¿Conoce los lugares de interés turístico y cultural del municipio?

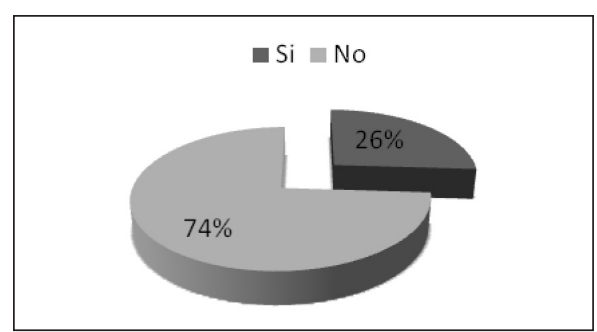

Grafico 2. ¿Considera que los recursos patrimoniales están bien gestionados?

Fuente: Elaboración propia.

En el gráfico 3 profundizamos un poco más en la percepción de la población sobre la gestión de los recursos patrimoniales del municipio, preguntando cómo perciben la evolución en la gestión de los recursos, sólo un $16 \%$ de los encuestados indican que la gestión ha mejorado, mientras que el $60 \%$ observan que sigue igual y el $24 \%$ opinan que ha empeorado.

Pero, ¿cómo valora la población de Sant Joan la iniciativa de crear un Centro de Interpretación del Patrimonio? Como refleja el gráfico 4, el 82\% de los individuos entrevistados, dan puntuaciones positivas acerca de la repercusión que consideran que puede tener el proyecto -tanto para la localidad como para su entorno-, mientras que el 18\% restante, o afirman no tener opinión al respecto o tener una opinión negativa acerca de este proyecto.

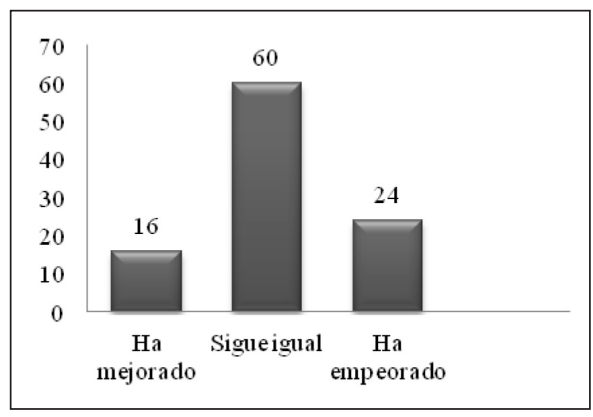

Grafico 3. ¿Cómo ha evolucionado la gestión de los recursos en los últimos años?

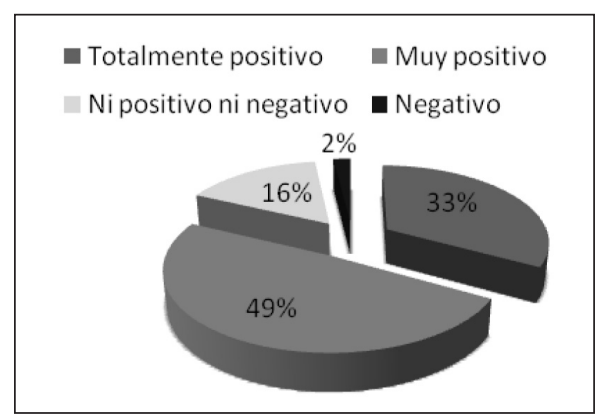

Gráfico 4. ¿Cómo valora la iniciativa de crear un Centro de Interpretación en el municipio?

Fuente: Elaboración propia. 
El patrimonio cultural es un rico legado que conservamos del pasado, si es lo suficientemente amplio e importante, debe ser uno de los elementos a tener en cuenta en lo que al desarrollo local se refiere. Así, el patrimonio cultural y su buena gestión nos deben llevar al aprovechamiento del mismo en su dimensión palpable, el turismo, para ser motor del desarrollo de la sociedad local (del Bien Sánchez, 2010).

Los Santjoaners apoyan este proyecto pues, tal como podemos ver en el gráfico 5, consideran que tendría una repercusión positiva para el municipio y la comarca, y le otorgan una puntuación media de 8,26 puntos sobre 10. Además, no hay que olvidar que sólo un $4 \%$ de los encuestados consideran que el proyecto tendría repercusiones negativas para el municipio y su comarca, y le otorgan una nota inferior al 5.

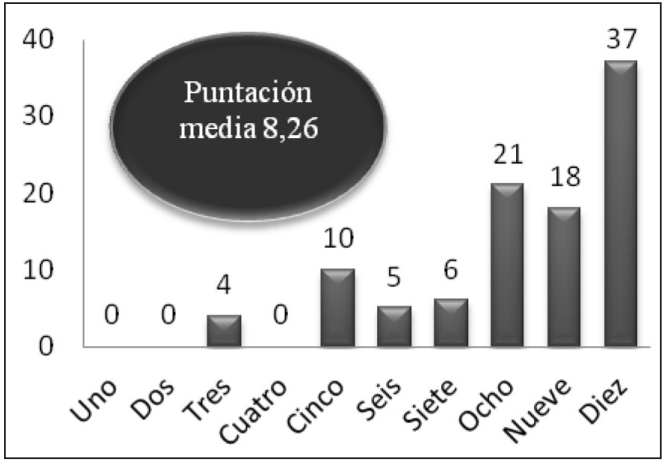

Gráfico 5. Puntúe de 0 a 10 la repercusión de este proyecto en el municipio.

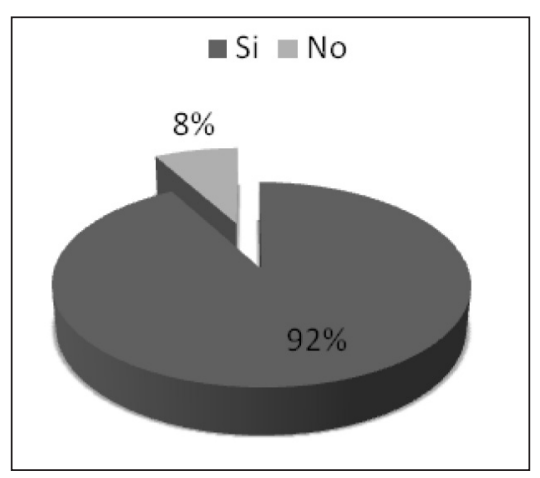

Gráfico 6. ¿Considera que el proyecto favorecerá al municipio de Sant Joan?

Fuente: Elaboración propia.

Para concluir, al preguntar si consideran que este proyecto va a favorecer a la localidad de Sant Joan, un total de nueve de cada diez ciudadanos santjoaners afirman que el proyecto va a beneficiar al municipio, tal como se muestra en el gráfico 6.

Entre los argumentos positivos que dan los individuos entrevistados a esta pregunta, reiteran que este proyecto va a impulsar la localidad gracias a "la creación de puestos de trabajo" y "al impulso que le dará al turismo", argumento señalado por la mayoría de los individuos encuestados.

\section{EL CENTRO DE INTERPRETACIÓN}

El Centro de Interpretación se quiere consolidar como sitio de referencia y recuperación de patrimonio, con la única idea de unificar en un solo lugar, todos los recursos turísticos, medioambientales, culturales, gastronómicos y patrimoniales que son demandados por los visitantes y por la población del municipio. 
De esta forma, se busca crear un lugar que nos permita viajar al pasado y dejar que los monumentos nos hablen de las experiencias vividas y de las maneras de entender la forma de vida en esta época, gracias a un entramado de actividades donde se combine historia, cultura y nuevas tecnologías.

Si protegemos el patrimonio, protegemos la identidad local y con ello las raíces y memoria de los ancestros del municipio.

Según las declaraciones de la UNESCO en la Convención de 1972 para la Protección del Mundo Cultural y el Patrimonio Natural «se manifestó que la herencia cultural y natural están amenazadas por la destrucción producida no sólo por las causas tradicionales de deterioro, sino por los cambios en las condiciones sociales y económicas que agravan la situación con fenómenos de destrucción» (Camarero Izquierdo, C., Garrido Samaniego, M. J., 2004).

Asimismo, se puede observar que la mayoría del escaso patrimonio de Sant Joan, a pesar de estar catalogado como Bien de Interés Cultural o como Bien de Relevancia Local, está sentenciado a una inminente desaparición, razón por la cual surge la necesidad de contribuir a que la sociedad conozca la identidad cultural y se implique en la promoción del patrimonio y así conservar y difundir el legado de los antepasados.

Los museos han ido evolucionando a lo largo de los años y se han creado ramas paralelas a ellos como son los centros de interpretación. Los dos albergan diferentes objetos y forman un conjunto de colecciones que se exponen para el disfrute y engrandecimiento del conocimiento del público, pero también conservan objetos en sus almacenes para restaurarlos y conservarlos.

El Centro de Interpretación se ubicará en la Finca de Pedro José situada junto a la nueva biblioteca municipal, en la Rambla de la Libertad. Muy cerca, el futuro Museo Fernando Soria, ubicado en la Casa del Reloj. Con estos tres edificios, se conseguirá formar un eje de encuentro socio-cultural dinámico y cercano, lo que sin duda, constituirá para el municipio un espacio de desarrollo turístico.

Elementos clave del Centro

La combinación de nuevas tecnologías, actividades didácticas y talleres serán las aliadas para dar a conocer a los visitantes cuestiones tales como ¿Por qué surge la idea de crear este Centro? ¿Por qué es importante conocer el pasado y las raíces de un pueblo? ¿Por qué ya se ha dejado de utilizar ciertas prácticas y costumbres?

Actualmente internet es una herramienta fundamental, una oportunidad de desarrollo para el turismo de interior y una de las más eficientes, además de facilitar el acceso a la información deseada. La reciente implantación de los códigos $\mathrm{QR}^{2}$ en las principales calles del municipio y en varios edificios y

2 El código QR es una imagen bidimensional que permite almacenar gran cantidad de información y contenidos que reduce el margen de error y la pérdida de datos. 
elementos patrimoniales ofrece la posibilidad de convertir el municipio en un territorio inteligente, pero para desarrollar este sistema, necesitamos implementar los siguientes aspectos clave:

Social: los medios sociales nos permiten la unión de personas y destinos a través de las redes sociales:

Local: la clave en el mundo globalizado es el elemento local y toda la información que gira a su alrededor.

Móvil: a través de los dispositivos móviles, las personas tienen la posibilidad y la capacidad de unir las dos cuestiones anteriormente nombradas: localizarse en un punto y momento deseado y a su vez compartirlo en la Red Social a la cual pertenecen.

Los Códigos QR permiten unir estos tres elementos, que llamamos So Lo $\mathrm{Mo}^{3}$, y presentarlos en un mismo recurso. La utilización de estos códigos es muy diversa, son de bajo coste, muy fáciles de utilizar, almacenan gran cantidad de información y permiten la interactuación de los usuarios entre ellos. Su tamaño es bastante reducido, por lo que se pueden incorporar a las colecciones sin alterar la apariencia de la exposición y aportar un valor añadido a la misma. Permiten transmitir información tanto en formato texto como en formato digital, audio o video y proporcionar al público una gran cantidad de datos acerca del municipio. Lo podemos ver a continuación,

\section{Cuadro 4. Código QR con información acerca del Centro de Interpretación}

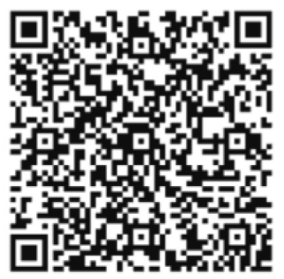

Fuente: Elaboración propia.

La metodología web 2.0 es un aspecto cada vez más decisivo para la demanda turística. Los comentarios de los internautas en internet sobre las experiencias vividas en un destino turístico o en una determinada empresa son un factor indispensable para la comercialización turística, pues nos fiamos más de las experiencias de otra gente que de lo que nos diga el propio gestor o propietario de establecimiento.

Por tanto, la introducción de las nuevas tecnologías al Centro de Interpretación hará posible la recuperación de parte del patrimonio que por diversas

3 Clase impartida por D. Gersón Beltrán en el curso de Territorios Innovadores en Contextos de Cambio, en Villena el 10 de julio de 2012. 
razones ya no existe. También se tiene previsto incorporar la Realidad Aumentada, tecnología totalmente nueva que une la realidad y lo virtual, es decir, es un entorno real fusionado con un entorno virtual. Con los dispositivos móviles y tabletas, los visitantes tendrán la posibilidad de visualizar los resultados obtenidos gracias a estos nuevos avances tecnológicos que nos ofrecen tanto los Códigos QR como la Realidad Aumentada.

La Realidad Aumentada es una potente y eficaz herramienta para divulgar y dar a conocer el patrimonio histórico y cultural de la ciudad, enriqueciendo y dando un valor añadido a toda la información que queremos transmitir.

En el cuadro siguiente podemos entender mejor qué es la Realidad Aumentada:

Cuadro 5. ¿Qué es la Realidad Aumentada?
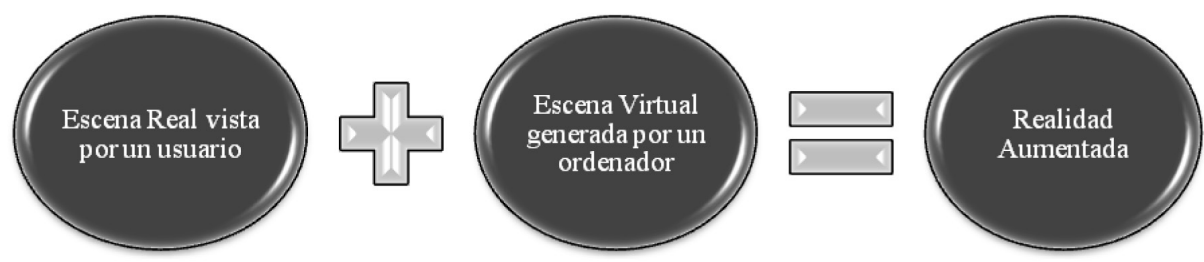

Fuente: Elaboración propia.

Begay y Dale afirman que «sólo se recuerda un $10 \%$ de lo que se lee, con la comunicación multimedia se consigue retener hasta un 50\%, pero el verdadero aprendizaje se consigue con la práctica y la interacción...» Es el llamado cono de la experiencia. Gracias a ello, se hace hincapié en la nueva manera de aprender del pasado con tecnología del presente ${ }^{4}$.

\subsection{Interior del centro y equipamientos}

Como ya hemos comentado, vamos a dotar el Centro con dos tipos de equipamientos que se utilizaran de forma conjunta. Por una parte tendremos los expositores tradicionales como son maquetas, dioramas, vitrinas y cartelas donde se expondrán los objetos que han sido donados y recuperados.

Por otra parte, la tecnológica, como son las pantallas táctiles, proyectores, Códigos QR y Realidad Aumentada, donde nuestros visitantes podrán disfrutar de un recorrido diferente e imborrable gracias a la tecnología implementada en nuestro Centro a través de maquetas virtuales, nuestros usuarios tendrán la posibilidad de descubrir más a fondo la historia del monumento visualizado, su uso en la época, o incluso el material con que se construyó.

4 Más información en http://mediamusea.com/category/difusion/ (Última consulta 16-092012). 
Llegados a este punto, debemos tener en cuenta de qué manera programar los códigos y proporcionar un volumen de información equilibrado y adecuado para la gran mayoría de los visitantes del Centro. Si bien es verdad, que existen segmentos de la población que no conocen o no tienen acceso a las nuevas tecnologías, nuestro propósito es llegar a todo tipo de público y ofrecerle un servicio informativo agradable, fácil y llamativo, razón por la cual hemos optado por combinar las nuevas tecnologías con las tradicionales.

También queremos implantar un sistema donde los personajes más destacables de la historia del municipio nos cuenten de forma virtual historias, anécdotas y tradiciones de nuestros antepasados. Para ello, queremos contar con la colaboración de una de las personas más conocedora de estas costumbres como es D. Isidro Buades, cronista del municipio, que nos puede proporcionar mucha información y ayudar a recrear momentos importantes de la historia de Sant Joan. Vamos a utilizar la tecnología holográfica en 3D con movimiento, con lo que no será necesario que la persona que da la conferencia sobre el patrimonio esté presente, en este caso D. Isidro Buades.

En la sala de proyecciones, sería interesante recrear algunas de las incursiones piratas del siglo XVI en nuestras tierras contadas por el temible pirata Dragut. Este terrible corsario turco nos adentrará en su mundo piratesco y gracias a él conoceremos la peor incursión jamás contada en nuestras tierras. Esta misma representación se podría proyectar en los exteriores de las torres, siempre que sus dueños estén de acuerdo o se restaure Torre Ansaldo, -la única torre de carácter público-, y organizar veladas veraniegas amenizadas con un espectáculo de luz y sonido. Esto sería, sin duda, una buena forma de despertar el interés por las torres de defensa.

\section{Conclusiones}

Sant Joan cuenta con una gran cantidad de patrimonio cultural disperso por la localidad. Para consolidarse como destino turístico es sumamente importante que el municipio revalorice su patrimonio histórico y consiga que se convierta en un punto de interés turístico que lleve a un aumento de estancias y consumo en el municipio.

Tal como lo hemos explicado hasta ahora, nuestro objetivo es implementar un proyecto de desarrollo turístico y cultural plasmado en el Centro de Interpretación, así como recuperar, conservar y proteger esos elementos patrimoniales que se encuentran actualmente en desuso y ruinosos. Las dos vertientes más importantes de este proyecto son pues la recuperación y la puesta en valor del patrimonio.

Estos dos objetivos están interrelacionados y en las condiciones actuales no se puede plantear la recuperación del patrimonio sin darle una utilización práctica y económica. Por tanto, con este proyecto y con el aumento de visi- 
tantes al pueblo, conseguiremos este objetivo en la medida que generará unos ingresos económicos indirectos para el municipio que nos permitirán mejorar y conservar el patrimonio así como su difusión.

En un estudio realizado de forma paralela se comprueba que la demanda turística ha cambiado con el paso del tiempo. Los visitantes buscan otro tipo de turismo, dejando de lado el tradicional turismo de sol y playa y dando paso a otro tipo de actividades, entre ellos, el turismo cultural. Por tanto, este proyecto se integra a estas nuevas tendencias de modalidad turística.

$\mathrm{Si}$ analizamos los diferentes datos desde un enfoque metódico como se recoge en el análisis DAFO, llegamos a la conclusión de que la planificación y organización de las diferentes etapas del proyecto, así como, los medios que tenemos a nuestro alcance tales como, recursos patrimoniales, nuevas tecnologías y la posibilidad de ubicar el Centro de Interpretación en una casa señorial que es parte de la riqueza del municipio, nos permitirán implementar un proyecto sostenible y beneficioso para el municipio, sus habitantes y la sociedad en general. A parte de las tareas que se deben llevar a cabo para poner en funcionamiento el Centro, se prevén unas acciones de promoción activa y sostenida a través de redes sociales y medios convencionales con el objetivo de difundir el producto hacia cada uno de los segmentos de la población y así adaptarlo a las posibles modificaciones de la demanda. De esta forma se conseguirá instaurar una política interactiva entre el visitante y la gestión de las señas culturales del municipio.

Esta fuerte apuesta por las TICs -Tecnología de la Información y las Comunicaciones- en la divulgación del patrimonio cultural nos permitirá adaptar nuestra política de comunicación a los diferentes públicos potenciales y captar un público más amplio, atraído por el lado moderno e innovador.

En la mayoría de los casos, el turista cultural planifica él mismo su viaje a través de internet, decide qué quiere visitar y cuándo lo quiere hacer. Por eso pensamos que los medios tecnológicos son una herramienta de promoción muy eficaz y adecuada para darle a conocer la evolución de nuestro proyecto y las diferentes actividades que se realicen y así multiplicar el grado de interés por nuestro municipio.

En cuanto a los habitantes del municipio, pensamos que el uso de las redes sociales puede ser efectivo siempre y cuando se consiga despertar y fomentar un grado de interés alto relativo a las actividades que surjan en torno al municipio. Somos conscientes de que dicho grado de interés no existe actualmente entre la población. No obstante, la creación del Centro de Interpretación reunirá todo ese patrimonio olvidado, lejano y desaparecido en un solo lugar, al alcance de todos. Tanto los mayores como los pequeños podrán disfrutar del material presentado de forma dinámica, diferente, divulgativa y lúdica, que les hará viajar en el tiempo sin tener que desplazarse in situ a cada uno de los lugares. 
Para finalizar, consideramos que la aplicación de este proyecto es de interés público y constituye sin duda una gran mejora en el nivel de calidad de los servicios ofertados por el municipio, tanto para los turistas como para los habitantes del lugar. Presenta la doble ventaja de potenciar el interés por la historia y las costumbres de nuestro pueblo y permite a la par rehabilitar el patrimonio cultural en desuso.

BiBLIOGRAFÍA

AA.VV. Ayuntamiento de Alicante (2007): Plan especial de protección y conservación de las torres de la Huerta de Alicante".

AA.VV. Ayuntamiento de Sant Joan d'Alacant (2010): Catálogo de bienes y espacios protegidos.

AA.VV. Cámara de Comercio de Alicante, (2012): Plan de Acción Comercial de Sant Joan d'Alacant.

ACAME POVEDA, V. (1999): «Historia de San Juan: La Condomina», Revista de los Comerciantes de San Juan.

BENDICHO, V. C. (1991): Crónica de la Muy Ilustre, Noble y Leal ciudad de Alicante, 1640, Cap, XIII, pág. 187. Ed. Ayuntamiento de Alicante.

CAMARERO IZQUIERDO, C.; y GARRIDO SAMANIEGO, M. J. (2004): Marketing del patrimonio cultural. Ed. Pirámide. Madrid.

CAMPELLO QUEREDA, A. (2002): Las torres de defensa de la huerta de Alicante.

CHEREM GABRIEL, J. (1977): "The Professional Interpreter: Agent of an Awakening Giant, Journal of Interpretation 2". USA en MORALES J. Guía Práctica para la Interpretación del Patrimonio.

DEL BIEN SÁNCHEZ, T. (2010): Gestión del Patrimonio Cultural en la ciudad de Toro: Casos prácticos y alternativas.

MARTÍNEZ PUCHE, A. (2009): Desarrollo local, recursos culturales y planes de dinamización de producto turístico en los espacios de interior. Análisis de políticas y escenarios futuros. III Fórum de Turismo de la Comunidad Valenciana.

MARTÍNEZ PUCHE, A. (2011): "Innovación Territorial", Calvo, R et alii, (coord.). Nuevos escenarios para el desarrollo local: políticas de empleo, innovación y responsabilidad territorial Col.lecció vuit fulles.

MAZÓN, T. (2001): Sociología del Turismo. Ed. Centro de Estudios Ramón Areces.

MOLINA, S., (1982): Ecología y Turismo. Editorial Trillas, México en MORALES J. Guía Práctica para la Interpretación del Patrimonio.

MORALES MIRANDA, J. (2001): Guía Práctica para la interpretación del Patrimonio. El arte de acercar el legado natural y cultural al público visitante. 
MOSCARDO, G.; y PHILIP L. PEARCE (1988): Using Tourism and Heritage Interpretation to Revive a Dying Town. Heritage Communicator 2(2):5-8. Canadá en MORALES J. Guía Práctica para la Interpretación del Patrimonio.

PEART, B., (1980): An application of the Foley and Keith Objectives Framework to Interpretation Activities. Journal of Interpretation 5(2):6-9 USA en MORALES J. Guía Práctica para la Interpretación del Patrimonio.

RUIZ TORRES, D., (2011): «Realidad Aumentada, Educación y Museos». Revista Icono 14, Año 9 vol. 2.

SÁNCHEZ BUADES, M. y SALA SEVA F. (1978): Resumen Histórico de la Villa de San Juan de Alicante, ed. Alicante.

UZZELL, D. (1989): The Natural and Built Environment. En: D. Uzzell (ed.), Heritage Interpretation, Vol. 1, Belhaven Press, London en MORALES J. Guía Práctica para la Interpretación del Patrimonio.

VERA REBOLLO, F. (1997): Análisis territorial del turismo. Ed. Ariel. Barcelona. 